\title{
Venom immunotherapy: pitfalls and open questions
}

\author{
"Despite all the new developments in the diagnosis of insect \\ venom allergies, there still remain many unmet needs in the diagnosis \\ and treatment of insect sting hypersensitivity."
}

\section{KEYWORDS: ACE inhibitor $\approx$ allergy $\approx$ anaphylaxis $\approx$ basophil activation test $\approx$ bee $\approx$ IgE * insect hypersensitivity $\approx$ risk factors " tryptase " venom " venom immunotherapy}

Depending on the climate, $56.6-94.5 \%$ of the general adult population have been stung by a hymenoptera species at least once in their lives [1]. The rate of self-reported systemic sting reactions (SSRs) in European epidemiological studies ranges from 0.3 to $8.9 \%$ while large local reactions (LLRs) range from $2.4-26.4 \%$ [1]. There is, however, to date no existing parameter that enables clinicians to predict who will have a future reaction and whether it will be a LLR or SSR. Recently, Bilo and Bonifazi reviewed in Immunotherapy that insect sting allergy and venom immunotherapy (VIT) remains an excellent model for the study of anaphylaxis and immune tolerance, and that VIT is the most effective form of specific immunotherapy [2]. Indications as well as contraindications, the natural history and risk factors, selection of venoms, treatment protocols, side effects, monitoring and duration of VIT, are well characterized by studies, with the daily practice of VIT following unequivocal guidelines [3] and practive parameter recommendations [4].

\section{"Many treatment protocols for VIT induction have been designed recently, promising to be faster, safer or more convenient to the patient."}

Indeed, VIT is extremely effective in reducing the risk of a subsequent SSR from an insect sting to less than 5\%. Some studies demonstrate that the side effects of VIT are even less frequent than those caused by subcutaneous immunotherapy for inhalant allergens [5]. VIT, being a treatment modality only for selected allergy centers a few decades ago, has thus become a standard procedure in the allergists office in many countries. However, many doctors and patients are not aware of the fact that, despite considerable progress in the past few years, much still remains to be accomplished and several important questions unanswered. This article summarizes the many open questions regarding the diagnosis of insect venom allergy and in particular its treatment.

The indication for VIT seems unequivocal, such as a history of SSR, a positive venom skin- or specific IgE test, knowledge of the natural history and established risk factors for a severe outcome [2]. Nevertheless, the risk of a future allergic reaction can only be expressed statistically, since none of the available tests are able to accurately predict the outcome of the next sting [5]. There is no positive correlation between the severity of a previous sting reaction and any specific test. Moreover, even a negative test result in patients with a clear history of a SSR does not rule out a future SSR. Although VIT is not usually recommended in patients with LLRs, physicians may consider it as a treatment option when confronted with extremely anxious patients with a health-related quality of life impairment; this anxiety is understandable since up to $7 \%$ of patients with LLRs might eventually develop SSRs to subsequent stings [6,7]. The same applies to children with only mild (i.e., cutaneous) SSRs, where the chance of having another SSR when restung is $13 \%$, patients who the administration of self-injectable adrenalin might be a problem and parental anxiety an additional motive [7]. Advantages of VIT in subjects with severe SSRs, increased baseline tryptase levels, cutaneous and/or systemic mastocytosis, elderly, cardiopathic patients, taking $\beta$-blockers and/or angiotensin-converting enzyme inhibitors, might outweigh the potential negative effects, despite all these factors being in principle contraindications for specific immunotherapy.

The selection of venoms is mostly hampered by the frequent cross-reactivity and double positivity of the seemingly limited differentiation tests currently available [8]. At present,

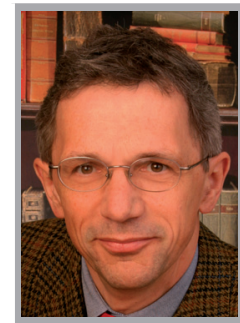

Werner Aberer

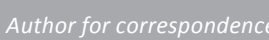
Department of Dermatology, Medical University of Graz, Auenbruggerplatz 8, 8036-Graz, Austria

Tel.: +4331638513925

Fax: +4331638513782 werner.aberer@medunigraz.at

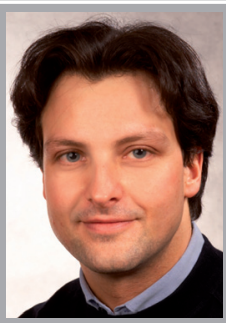

Gunter Sturm Department of Dermatology, Medical University of Graz, Auenbruggerplatz 8, 8036-Graz, Austria 
recombinant allergens are incompletely available and component-resolved diagnosis therefore closes the gap only in part [9].

\section{"...the goal of VIT is primarily to prevent life-threatening reactions. And it does."}

Many treatment protocols for VIT induction have been designed recently, promising to be faster, safer or more convenient to the patient [10]. However, the selection as to which protocol is best for the individual patient remains undefined, since criteria such as safety, side effects, individual risk factors and the personal situation of the patient all have to be taken into account. Risk factors for VIT-induced SSRs must be taken into account and patients with one or more risk factors should be treated and monitored with special care. Recent recommendations, however, to automatically double the venom dose in patients with mastocytosis are difficult to follow in patients with a history of severe SSR or cardiovascular disease, although it might be important especially in these patients [11]. The same is true for patients with autoimmune disease, where an acute worsening of the disease, as well as a possible reduced effectiveness, should be taken into consideration. The efficacy of VIT is generally taken as being excellent; but VIT is not completely effective. Even the high dose of 300-400 $\mu \mathrm{g}$ of mixed vespid venoms, which most American patients receive as maintenance dose, provides only $98 \%$ protection. In Europe, where the recommended dose in children and adults for bee and wasp venom allergy is $100 \mu \mathrm{g}$, the protection rate of honeybee VIT is $77-94 \%$, which is less than that offered by vespid VIT (91-100\%) [12]. The question why VIT with honeybee venom is less effective than that with vespid venom is still a debated issue. Generally, risk factors for treatment failures or reduced effectiveness are numerous, but frequently not taken into consideration in daily practice.

Venom immunotherapy monitoring during active VIT serves two purposes: to check whether the patient is protected against a field sting and whether VIT might be abandoned. However, there is currently no reliable method to tell whether the patient will or will not react, since repeated skin or blood (e.g., venom-specific IgG, IgG4 and basophil activation test) testing as recommended by several authors and guidelines does not give an absolute answer and the tests are mostly performed because of scientific, financial or other interests. And even a sting challenge test identifies only those who are not protected but does not, when being negative, allow telling the patient to be protected [13].
Annual appointments are, however, essential in order to keep up the compliance of our patients. Regarding the duration of VIT, our knowledge is based more on experience than evidence. Taking into consideration the many risk factors for the recurrence of a systemic reaction after discontinuing VIT, such as honeybee venom allergy, being of adult age, severe pretreatment SSRs, SSRs during VIT to treatment injection or restings, mastocytosis, and elevated baseline tryptase levels, one might question the common policy of stopping VIT after 3-5 years. Negative skin and specific $\mathrm{IgE}$ tests might convince doctors and patients that no reaction will occur upon a future field sting. However, if some authors postulate that patients should still carry an emergency kit after ending VIT [14], one might question our faith into the reliability of VIT or negative skin tests. The same is true regarding the recommendation to continue monitoring regularly [15]. The recommendation from other authors of the European guidelines to consider longer-term (or even life-long) treatment in patients with a higher risk of severe SSR (e.g., older age, history of severe previous SSR, elevated basal serum tryptase, mastocytosis or use of $\beta$-blockers), generalized allergic reactions to stings or to immunotherapy injections during VIT, and highly exposed patients (such as beekeepers and their immediate family members) might follow some logic. However, to perform long-term VIT in patients at a low risk of relapse for personal safety and improved quality of life (e.g., limitation of activity and anxiety about unexpected stings) as practiced in some institutions does not seem to be justified.

There is general agreement that the goal of VIT is primarily to prevent life-threatening reactions. And it does. However, both efficacy and safety of VIT are not as good as is often claimed, especially with bee venom, where $10-20 \%$ of treated patients develop some, mostly mild, systemic allergic symptoms when restung during VIT, and 20-30\% develop systemic allergic side effects, especially during rush and ultrarush protocols for dose increase [16]. A secondary benefit is that VIT might alleviate anxiety related to insect stings; we as doctors might have neglected this aspect too long. Nevertheless, we should also consider whether our policy to treat for a long duration may actually induce anxiety and make our patients more and concerned about stings, in addition to the efforts and costs for our patients and the social system.

Studies in emergency departments show the need for better recognition and prevention of insect sting-induced anaphylaxis. Patients treated 
for allergic reactions to stings need better counseling on avoidance, use of epinephrine injectors and the need for allergy evaluation and treatment [4]. A recent telephone survey has shown that even a considerable proportion of high-risk patients are neither equipped with epinephrine injectors nor informed regarding VIT [17]. Whether this is due to noncompliance of the affected individuals or due to poor information by the emergency doctors who were treating the reactions was not evaluated.

Despite all the new developments in the diagnosis of insect venom allergies, there still remain many unmet needs in the diagnosis and treatment of insect sting hypersensitivity [4]. Tests to improved diagnostic accuracy are undergoing evaluation in studies. Recombinant allergens or epitopes are hoped to provide improved predictive values for the occurence and type of potential future reaction in patients. Similarly, there is need for a better predictor of relapse after stopping VIT, a study of discontinuation after $12-15$ years in 'high-risk' patients with negative skin test responses, and perhaps most of all, an effective screening test to detect the $50 \%$ of fatal sting reactors who die on their first reaction and therefore cannot be prevented by current standards of testing [4]. A dramatic figure yet not surprising cognizant of the $17 \%$ chance of a SSR to a future sting in the absence of a history of a sting-induced allergic reaction, but sensitization through this asymptomatic sting [18].

Recently, the first comprehensive audit of the diagnosis and management of hymenoptera venom allergic patients in the UK revealed a wide variation in practice and suggested the need for the development of better educational programs for specialists and trainees involved in the management of the patients in question [19]; quite a surprise when considering the vast amount of scientific literature published in recent years.

\section{Financial \& competing interests disclosure}

The authors have no relevant affiliations or financial involvement with any organization or entity with a financial interest in or financial conflict with the subject matter or materials discussed in the manuscript. This includes employment, consultancies, honoraria, stock ownership or options, expert testimony, grants or patents received or pending, or royalties.

No writing assistance was utilized in the production of this manuscript.

\section{Bibliography}

1 Bilo MB, Bonifazi F. The natural history and epidemiology of insect venom allergy: clinical implications. Clin. Exp. Allergy 39, 1467-1476 (2009).

2 Bilo BM, Bonifazi F. Hymenoptera venom immunotherapy. Immunotherapy 3, 229-246 (2011).

3 Bilo BM, Rueff F, Mosbech H, Bonifazi F, Oude-Elberink JN. Diagnosis of hymenoptera venom allergy. Allergy 60, 1339-1349 (2005).

4 Golden DB, Moffitt J, Nicklas RA et al. Stinging insect hypersensitivity: a practice parameter update 2011. J. Allergy Clin. Immunol. 127, 852-854 (2011).

5 Aberer W, Sturm G. Bees and wasps may be dangerous, but who is at risk? Int. Arch. Allergy Immunol. 153, 107-108 (2010).

6 Graft DF, Schuberth KC, Kagey-Sobotka A et al. A prospective study of the natural history of large local reactions after hymenoptera stings in children. J. Pediatr. 104, 664-668 (1984).

7 Golden DB, Kagey-Sobotka A, Norman PS, Hamilton RG, Lichtenstein LM. Outcomes of allergy to insect stings in children, with and without venom immunotherapy. $N$. Engl. J. Med. 351, 668-674 (2004).
8

Sturm GJ, Jin C, Kranzelbinder B et al. Inconsistent results of diagnostic tools hamper the differentiation between bee and vespid venom allergy. PLoS ONE 6, E20842 (2011).

9 Sturm GJ, Hemmer W, Hawranek T et al. Detection of $\operatorname{IgE}$ to recombinant Api $\mathrm{m} 1$ and rVes $v 5$ is valuable but not sufficient to distinguish bee from wasp venom allergy. J. Allergy Clin. Immunol. 128, 247-248 (2011).

10 Sturm G, Kranke B, Rudolph C, Aberer W. Rush hymenoptera venom immunotherapy: a safe and practical protocol for high-risk patients. J. Allergy Clin. Immunol. 110, 928-933 (2002).

11 Rueff F, Przybilla B, Bilo MB et al. Predictors of severe systemic anaphylactic reactions in patients with hymenoptera venom allergy: importance of baseline serum tryptase-a study of the European Academy of Allergology and Clinical Immunology Interest Group on Insect Venom Hypersensitivity. J. Allergy Clin. Immunol. 124, 1047-1054 (2009).

12 Bonifazi F, Jutel M, Bilo BM, Birnbaum J, Muller U. Prevention and treatment of hymenoptera venom allergy: guidelines for clinical practice. Allergy 60, 1459-1470 (2005).

13 Rueff F, Przybilla B, Muller U, Mosbech H. The sting challenge test in hymenoptera venom allergy. Position paper of the
Subcommittee on Insect Venom Allergy of the European Academy of Allergology and Clinical Immunology. Allergy 51, 216-225 (1996).

14 Rueff F, Przybilla B, Bilo MB et al. Predictors of side effects during the buildup phase of venom immunotherapy for hymenoptera venom allergy: the importance of baseline serum tryptase. J. Allergy Clin. Immunol. 126, 105-111 (2010).

15 Przybilla B, Rueff F. Hymenoptera venom allergy. J. Dtsch. Dermatol. Ges. 8. 114-129 (2010).

16 Muller UR. Recent developments and future strategies for immunotherapy of insect venom allergy. Curr. Opin. Allergy Clin. Immunol. 3 , 299-303 (2003)

17 Bokanovic D, Aberer W, Griesbacher A, Sturm GJ. Prevalence of hymenoptera venom allergy and poor adherence to immunotherapy in Austria. Allergy 66, 1395-1396 (2011).

18 Golden DB, Marsh DG, Freidhoff LR et al. Natural history of hymenoptera venom sensitivity in adults. J. Allergy Clin. Immunol. 100, 760-766 (1997).

19 Diwakar L, Noorani S, Huissoon AP et al. Practice of venom immunotherapy in the United Kingdom: a national audit and review of literature. Clin. Exp. Allergy 38, 1651-1658 (2008). 